\title{
Search for Heavy and Excited Leptons at LEP
}

\author{
B. Vachon ${ }^{\mathrm{a}}$

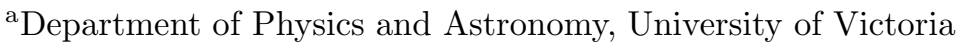 \\ P.O. Box 3055, Victoria, B.C., V8W 3P6, Canada.
}

\begin{abstract}
New particle searches are ongoing at the Large Electron Positron Collider (LEP) running yearly at increasing centre-of-mass energies. The searches for heavy stable and unstable leptons performed by the four LEP experiments (ALEPH, DELPHI, L3 and OPAL) are summarised, followed by a description of the searches for excited leptons. Experimental signatures and results, including the most recent data analysed, are presented.
\end{abstract}

\section{Physics Beyond the Standard Model}

Although very successful in describing experimental observations, the Standard Model (SM) of particle physics is however incomplete. It relies on many assumptions that cannot be explained within its framework. The search for physics beyond the SM is therefore an active field which takes on two different approaches.

One approach includes models such as Grand Unified Theories $\left(\mathrm{E}_{6}, \mathrm{SO}(10) \ldots\right)$ and supersymmetry (SUSY). Within this frame work, the known SM particles are regarded as being fundamental and point-like. Some of these models predict the existence of new fundamental particles such as heavy charged $\left(\mathrm{L}^{ \pm}\right)$and neutral $(\mathrm{N})$ leptons.

The second approach to physics beyond the SM assumes that known particles have substructure at a scale $\Lambda \gtrsim 1 \mathrm{TeV}$. Composite models could explain the number of fermion generations (or families) and the mass spectrum observed within the SM. Under the assumption of compositeness, it is natural to expect the presence of excited states of the SM fermions.

With the ongoing increase in energy and luminosity at the LEP collider, new particles could be within experimental reach. Various searches are performed by the four LEP experiments. This note reviews the searches for heavy and excited leptons. Experimental signatures and results of the search for heavy stable/long-lived and unstable leptons are presented. Then, a description of
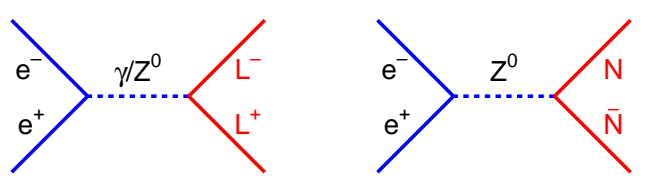

Figure 1. Feynman diagrams representing the pair production of heavy charged $\left(\mathrm{L}^{ \pm}\right)$and neutral $(\mathrm{N})$ leptons in $\mathrm{e}^{+} \mathrm{e}^{-}$collisions.

the searches for single and pair production of excited leptons follows. Unless stated, the results presented here include data taken in 1999 at a centre-of-mass energy up to $202 \mathrm{GeV}$, corresponding to an integrated luminosity of about $220 \mathrm{pb}^{-1}$ per experiment.

\section{Searches for Heavy Leptons}

Heavy leptons can be sequential, mirror or vector leptons[4]. Neutral heavy leptons could, in addition, be Dirac or Majorana particles.

The mixing between heavy and SM leptons is severely constrained by LEP1 data and lowenergy experiments [5]. Therefore, only the searches for pair produced heavy leptons are considered. Charged $\left(\mathrm{L}^{ \pm}\right)$and neutral $(\mathrm{N})$ leptons could be pair produced in $\mathrm{e}^{+} \mathrm{e}^{-}$collisions via the diagrams shown in Figure 1. Unstable leptons 
subsequently decay via charged (CC) or neutral (NC) current interactions (only CC decays are allowed for sequential leptons since flavour changing neutral current (FCNC) is forbidden within the SM). Charged current decays generally dominate for the mass range and different type of unstable leptons considered. Thus only final states consistent with $\mathrm{CC}$ decays are searched for.

\subsection{Heavy Unstable Charged Leptons}

Unstable charged leptons decay via $\mathrm{CC}$ to a $\mathrm{W}$ boson and a neutrino. The $\mathrm{W}$ boson in turn can decay either leptonically or hadronically.

$$
\begin{gathered}
\mathrm{e}^{+} \mathrm{e}^{-} \longrightarrow \mathrm{L}^{+} \mathrm{L}^{-} \\
\stackrel{L}{\longrightarrow} \nu_{\ell} \mathrm{W}^{-} \\
\stackrel{\bar{\nu}_{\ell} \mathrm{W}^{+}}{\longrightarrow} \stackrel{\mathrm{q} \overline{\mathrm{q}} \text { or } \ell^{-} \bar{\nu}_{\ell}}{\longrightarrow \mathrm{q} \overline{\mathrm{q}} \text { or } \ell^{+} \nu_{\ell}}
\end{gathered}
$$

The two experimental topologies considered are events with four jets and missing momentum (due to the presence of neutrinos from the heavy lepton CC decay) or events with two jets, one lepton and missing momentum. Final states where both $\mathrm{W}$ bosons decay leptonically are not considered due to the rather small W leptonic branching fraction. The efficiency of the different selections is of the order of $20 \%$ and the main background comes from hadronic and semi-leptonic $\mathrm{W}^{+} \mathrm{W}^{-}$ events.

\subsection{Heavy Unstable Neutral Leptons}

The following decays of heavy unstable neutral leptons are considered.

$$
\begin{aligned}
& \mathrm{e}^{+} \mathrm{e}^{-} \longrightarrow \mathrm{N} \overline{\mathrm{N}}
\end{aligned}
$$

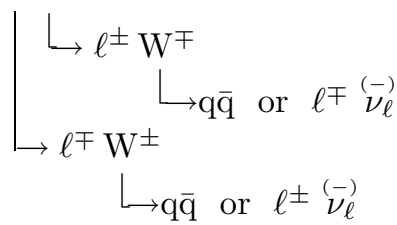

Candidate events contain either four jets and two isolated leptons or two jets, three leptons and missing momentum. Furthermore, because Majorana $\mathrm{N}$ can decay either to $\mathrm{W}^{+}$or $\mathrm{W}^{-}$, events

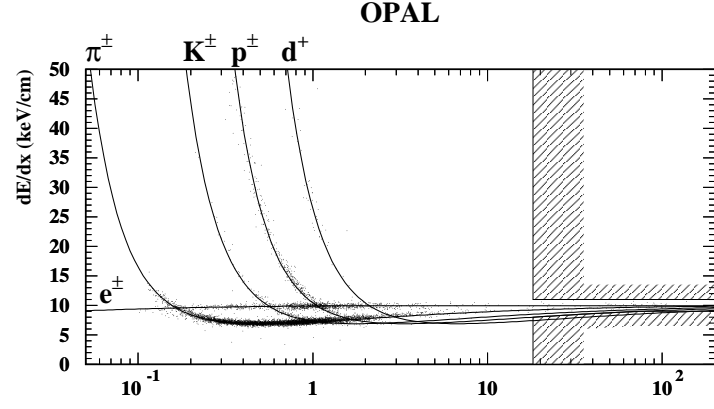

(a)

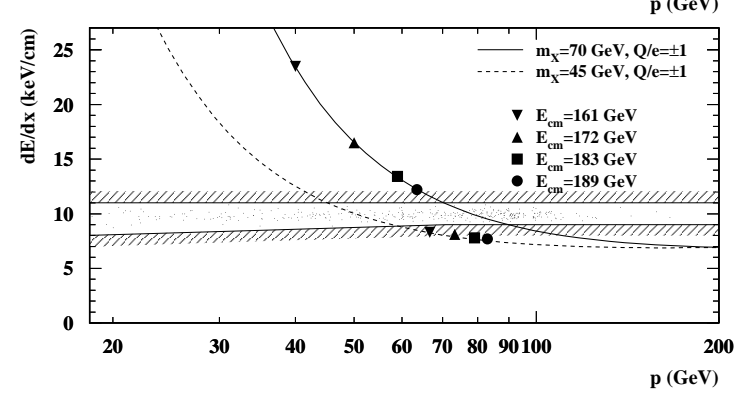

(b)

Figure 2. (a) Ionization energy loss $(\mathrm{dE} / \mathrm{dx})$ as a function of momentum (p) for different SM particles. The search regions are hatched. (b) Expanded view of the search regions. Theoretical predictions for different heavy long-lived particle are shown.

can contain leptons of the same charge. Therefore, to remain sensitive to Majorana N, no requirement on the sign of the isolated leptons is imposed. The efficiency of the different selections is about $35 \%$ and the main background for this search comes from multihadronic $q \bar{q}$ events and four-fermion processes $\ell \ell q q$ and qqqq.

\subsection{Heavy Stable/Long-Lived Leptons}

Heavy stable or long-lived leptons produce two back-to-back tracks. Both tracks are required to have an ionization energy loss $(\mathrm{dE} / \mathrm{dx})$ outside the range of SM particle. Figure 2 shows the $\mathrm{dE} / \mathrm{dx}$ distribution of SM particles as a function of momentum (p). The hatched area is the search region [6]. In the $\mathrm{dE} / \mathrm{dx}$ region overlapping with SM particles, heavy leptons are identified using 
Table 1

95\% CL lower mass limits for the searches of heavy sequential leptons.

\begin{tabular}{clcc}
\hline \multicolumn{2}{c}{ Channel } & OPAL & L3 \\
\hline $\mathrm{N} \rightarrow \mathrm{eW}$ & Dirac & 97.8 & 97.1 \\
& Majorana & 88.8 & 85.7 \\
$\mathrm{~N} \rightarrow \mu \mathrm{W}$ & Dirac & 97.1 & 97.0 \\
& Majorana & 87.7 & 88.0 \\
$\mathrm{~N} \rightarrow \tau \mathrm{W}$ & Dirac & 78.9 & 89.0 \\
& Majorana & 67.5 & 79.6 \\
\hline $\mathrm{L}^{ \pm} \rightarrow \nu_{\ell} \mathrm{W}^{ \pm}$ & $91.3^{\dagger}$ & 98.8 \\
\hline $\mathrm{L}^{ \pm}$stable/long-lived & 98.0 & $93.5^{\dagger}$ \\
\hline & data recorded up to $\sqrt{s}=189 \mathrm{GeV}$
\end{tabular}

kinematic properties of the event.

\subsection{Mass Limits}

In all searches for heavy leptons, the number of observed events agrees with the SM prediction [710]. Lower mass limits for heavy sequential leptons are calculated by different LEP experiments and presented in Table 1. Mass limits for Majorana neutral leptons are lower than for Dirac leptons because the Majorana production crosssection decreases more rapidly with mass. Mass limits for both charged and neutral vector leptons are approximately $100 \mathrm{GeV}[7]$. Vector leptons have a larger production cross-section than sequential and mirror leptons which explains the higher mass limits obtained.

\section{Searches for Excited Leptons}

The existence of excited leptons is severely constrained by g-2 precision measurements [11]. Generally, composite models [12,13] assume that both left and right-handed components of excited leptons form weak isodoublets. The coupling of excited leptons to a gauge boson $\left(\ell^{*} \ell^{*} \mathrm{~V}\right)$ is then purely vector. The single production crosssection and branching fractions of excited leptons are determined by the strength of the $\ell \ell^{*} \mathrm{~V}$ coupling. This interaction can be described by the following $\mathrm{SU}(2) \times \mathrm{U}(1)$ gauge invariant effective Lagrangian [12]

$$
\mathcal{L}_{\ell \ell^{*} V}=\frac{1}{2 \Lambda} \bar{\ell}^{*} \sigma^{\mu \nu}\left[g f \frac{\boldsymbol{\tau}}{2} \mathbf{W}_{\mu \nu}+g^{\prime} f^{\prime} \frac{Y}{2} B_{\mu \nu}\right] \ell_{\mathrm{L}}
$$

(a)

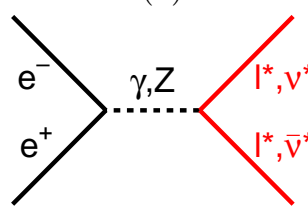

(b)

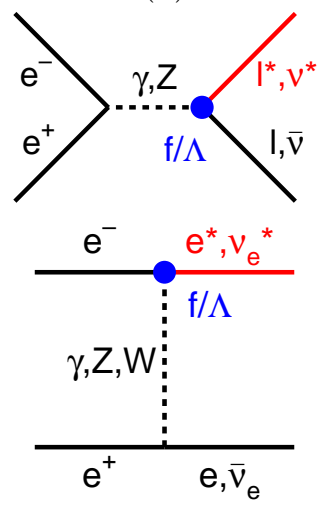

Figure 3. Dominant diagrams representing (a) pair and (b) single production of excited leptons. The vertices with a blob are suppressed by a factor of $f / \Lambda$.

where $\sigma^{\mu \nu}$ is the covariant bilinear tensor, $\boldsymbol{\tau}$ denotes the Pauli matrices, $Y$ is the weak hypercharge, $\mathbf{W}_{\mu \nu}$ and $B_{\mu \nu}$ represent the SM gauge field tensors and the couplings $g, g^{\prime}$ are the $\mathrm{SU}(2)$ and $\mathrm{U}(1)$ coupling constants of the SM. The parameter $\Lambda$ which has units of energy sets the compositeness scale. Finally, the strength of the $\ell \ell^{*} \mathrm{~V}$ coupling is governed by the constants $f$ and $f^{\prime}$. These constants can be interpreted as weight factors associated to the different gauge groups. The assignment $f=f^{\prime}$ or $f=-f^{\prime}$ is usually chosen such that the only free parameter (apart from the mass of the excited lepton) is $f / \Lambda$. Excited leptons can be singly or pair produced via the diagrams shown in Figure 3. They decay to SM leptons via the emission of $\gamma, \mathrm{Z}^{0}$ or $\mathrm{W}^{ \pm}$.

\subsection{Pair Production}

Pair produced excited leptons decaying photonically are identified by events containing two leptons and two photons $(\ell \ell \gamma \gamma, \nu \nu \gamma \gamma)$ in the final state. Excited leptons decaying via charged current produce the same topologies $(\nu \nu W W, \ell \ell W W)$ as heavy leptons and therefore the same analysis is used. In addition to these final states, the L3 Collaboration [14] search for 
Table 2

95\% CL lower mass limits of excited leptons.

\begin{tabular}{cccccccc}
\hline & \multicolumn{3}{c}{$f=f^{\prime}$} & \multicolumn{2}{c}{$f=-f^{\prime}$} & \multicolumn{2}{c}{ Coupl. Ind. } \\
\hline & L3 & OPAL & DELPHI & ALEPH & L3 & OPAL & L3 \\
\hline $\mathbf{e}^{*}$ & 100.1 & 100.1 & $94.1^{\dagger}$ & $94.3^{\dagger}$ & 96.2 & $91.3^{\dagger}$ & 96.0 \\
$\boldsymbol{\mu}^{*}$ & 100.3 & 100.1 & $94.1^{\dagger}$ & $94.3^{\dagger}$ & 96.2 & $91.3^{\dagger}$ & 96.2 \\
$\boldsymbol{\tau}^{*}$ & 99.9 & 100.0 & $93.3^{\dagger}$ & $94.3^{\dagger}$ & 96.2 & $91.3^{\dagger}$ & 94.9 \\
\hline $\boldsymbol{\nu}_{\mathbf{e}}^{*}$ & 99.3 & 99.5 & $93.6^{\dagger}$ & $94.2^{\dagger}$ & 99.5 & $94.3^{\dagger}$ & 98.5 \\
$\boldsymbol{\nu}_{\boldsymbol{\mu}}^{*}$ & 99.4 & 99.5 & $93.7^{\dagger}$ & $94.2^{\dagger}$ & 99.5 & $94.3^{\dagger}$ & 98.5 \\
$\boldsymbol{\nu}_{\boldsymbol{\tau}}^{*}$ & 93.9 & 91.9 & $89.5^{\dagger}$ & $94.2^{\dagger}$ & 99.4 & $94.3^{\dagger}$ & 92.7 \\
\hline
\end{tabular}

$\dagger$ limits calculated from data recorded up to $\sqrt{s}=189 \mathrm{GeV}$

mixed decay events where one excited lepton decays photonically and the other via $\mathrm{CC}\left(\mathrm{e}^{+} \mathrm{e}^{-} \rightarrow\right.$ $\left.\ell^{*} \ell^{*} \rightarrow \ell \gamma \nu \mathrm{W}\right)$. In all the different final states considered, no excess is found in the data. The 95\% CL lower mass limits $[9,10,14,16]$ of excited leptons are shown in Table 2.

\subsection{Single Production}

For single production searches, all possible excited lepton decays are consider which results in various possible final states. Typical event topologies include $\ell \ell \gamma, \nu \nu \gamma, \ell \nu W, \ell \ell Z$ and $\nu \nu Z$. If excited leptons exist, a peak should appear in the invariant mass distribution of the excited lepton decay products. Figure 4 shows the $\ell \gamma$ invariant mass for electron, muon and tau candidates. Typical mass resolutions are of the order of 0.3 to $1 \mathrm{GeV}$ for $\mathrm{e} \gamma$ and $\mu \gamma$, and around 1.5 to 2 $\mathrm{GeV}$ for $\tau \gamma$ events. No mass peak is observed in the data. Limits on the coupling over compositeness scale $(f / \Lambda)$ as a function of the excited lepton mass are calculated assuming $f=f^{\prime}$ and $f=-f^{\prime}[14,15,17]$. Example of these limits are shown in Figure 5 and 6.

\section{Summary}

With the increase in centre-of-mass energy at LEP, the search for heavy and excited leptons continues. The four LEP experiments have so far not observed any excess in the data consistent with the presence of new leptons. Using data collected as a centre-of-mass energy up to $202 \mathrm{GeV}$, 95\% lower mass limits for heavy and excited lep-

\section{OPAL Preliminary}

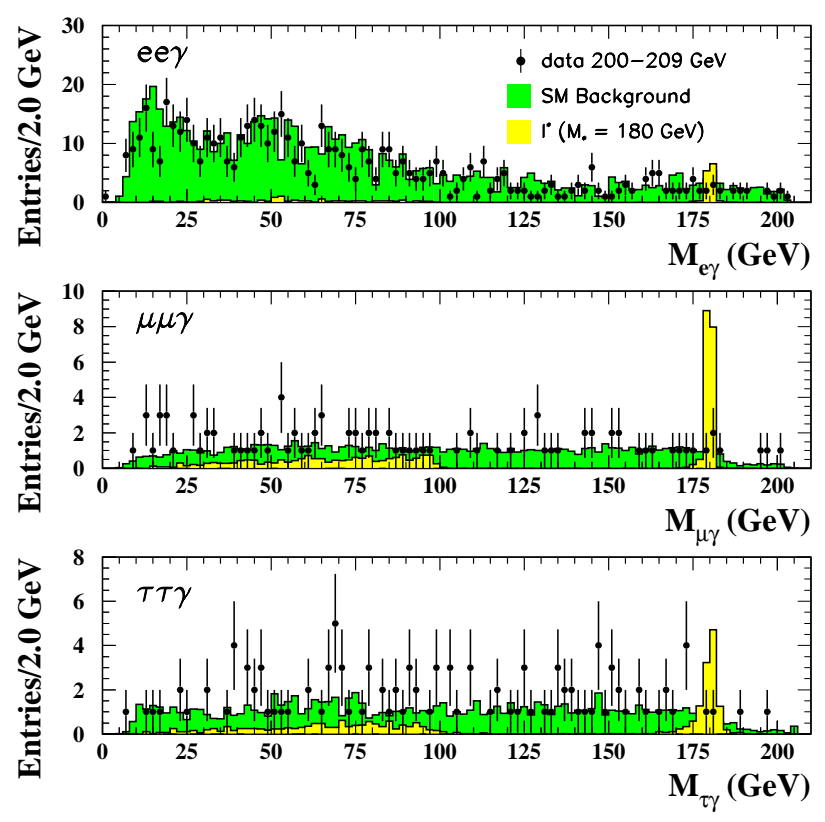

Figure 4. Reconstructed $\ell \gamma$ invariant mass for single production electron, muon and tau candidate events. There are two entries per event due to pairing ambiguity. The light grey histograms represent excited lepton events with a mass of $180 \mathrm{GeV}$ and normalised to a coupling over compositeness scale $(f / \Lambda)$ of $0.5 \mathrm{TeV}^{-1}$ for electron and $5 \mathrm{TeV}^{-1}$ for muon and tau. 

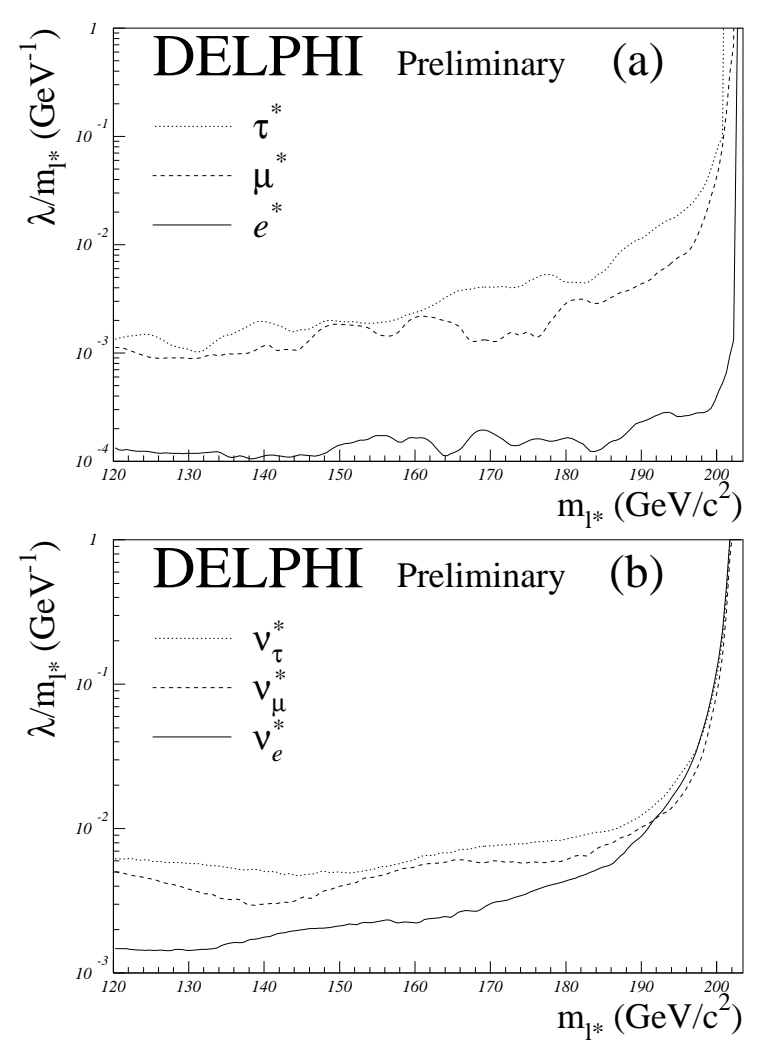

Figure 5. 95\% confidence level upper limits on the ratio of the coupling to compositeness scale as a function of the excited lepton mass, assuming $f=f^{\prime}$. The parameterisation used by the DELPHI Collaboration corresponds to $\lambda / \mathrm{m}_{\ell^{*}}=$ $f / \sqrt{2} \Lambda$. The regions above the curves are excluded.
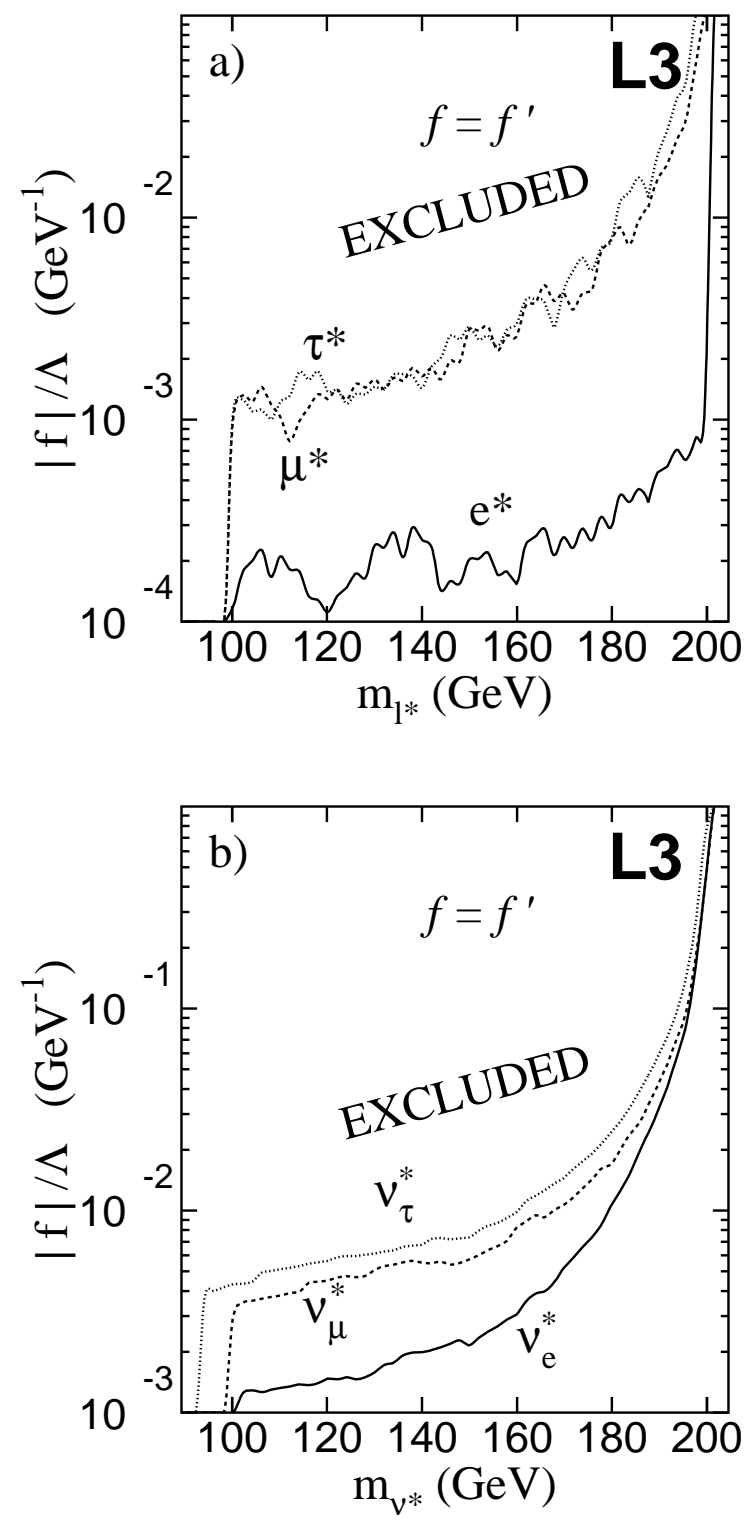

Figure 6. 95\% confidence level upper limits on the ratio of the coupling to compositeness scale, $f / \Lambda$, as a function of the excited lepton mass, assuming $f=f^{\prime}$. The regions above the curves are excluded. 
tons are calculated to be about 90 to $100 \mathrm{GeV}$. Furthermore, the search for singly produced excited leptons yields limits on $f / \Lambda$ as a function of mass. The ongoing increase in energy and integrated luminosity at LEP continues to open a new window for discovery.

\section{REFERENCES}

1. Super-Kamiokande Collaboration, D. Kielczewska for the collaboration, Nucl. Phys. Proc. Suppl. 81 (2000) 133-142;

Super-Kamiokande Collaboration, Y. Fukuda et al., Phys. Rev. Lett. 81 (1998) 1562-1567.

2. MACRO Collaboration, M. Spurio for the collaboration, Nucl. Phys. A663 (2000) 779-782.

3. M. Gell-Mann, P. Ramond and R. Slansky, Rev. Mod. Phys. 50 (1978) 721;

T. Yanagida, Phys. Rev. D20 (1979) 2986.

4. A. Djouadi, Z. Phys. C63 (1994) 317;

F. Boudjema, A. Djouadi and J.L. Kneur, Z. Phys. C57 (1993) 425.

5. D. London, "Exotic Fermions", Precision Tests of the Standard Model, 1993, ed. P.Langacher, World Scientific.

6. OPAL Collaboration, K. Ackerstaff et al., Phys. Lett. B433 (1998) 195-208.

7. L3 Collaboration, "Search for Exotic Unstable Heavy Leptons in $e^{+} e^{-}$Collisions at $\sqrt{s}=192-202$ GeV', L3 Note 2550, ICHEP 2000 Contributed Paper 501, June 2000.

8. L3 Collaboration, M. Acciarri et al., Phys. Lett. B462 (1999) 354-364.

9. OPAL Collaboration, "New Particle Searches in $e^{+} e^{-}$Collisions at $\sqrt{s}=189$ GeV', OPAL Physics Note PN370, November 1998.

10. OPAL Collaboration, "New Particle Searches in $e^{+} e^{-}$Collisions at $\sqrt{s}=192-202 \mathrm{GeV}$ ", OPAL Physics Note PN418, ICHEP 2000 Contributed Paper 185, November 1999.

11. S.J. Brodsky and S.D. Drell, Phys. Rev. D22 (1980) 2236

P. Mery, S.E. Moubarik, M. Perrottet and F.M. Renard, Z. Phys. C46 (1990) 229;

F.M. Renard, Phys. Lett. B116 (1982) 264;

J.I. Aranda, R. Martinez and O.A. Sampayo, Phys. Rev. D62 (2000) 013010.

12. F. Boudjema, A. Djouadi and J.L. Kneur, Z.
Phys. C57 (1993) 425.

13. K. Hagiwara, D. Zeppenfeld and S. Komamiya, Z. Phys. C29 (1985) 115.

14. L3 Collaboration, "Search for Excited Leptons at $\sqrt{s}=192-202$ GeV", L3 Note 2572, ICHEP 2000 Contributed Paper 498, June 2000.

15. OPAL Collaboration, "Search for Charged Excited Leptons with Photonic Decays in $e^{+} e^{-}$Collisions at $\sqrt{s}=189-202 \mathrm{GeV}$ at LEP', OPAL Physics Note PN434, ICHEP 2000 Contributed Paper 236, July 2000.

16. ALEPH Collaboration, "Search for Excited Leptons in $e^{+} e^{-}$Collisions at $\sqrt{s}=188.6$ GeV', ALEPH Note 99-068 CONF 99-042, EPS-HEP 99 Contributed Paper 7-419, June 1999;

DELPHI Collaboration, "Update on the Search for Excited Fermions at LEP", DELPHI Note 99-71 CONF 258, EPS-HEP 99 Contributed Paper 7-115, March 2000.

17. DELPHI Collaboration, "Update on the Search for Singly Produced Excited Leptons at LEP", DELPHI Note 2000-035 CONF 354, Moriond 2000 Contributed Paper, March 2000 . 